\title{
Emotional Intelligence Levels among Hearing-impaired and Visually Impaired Students in Jordan
}

\section{Akef Abdullah Al - Khateeb ${ }^{1}$ Wael Mohammad Alshurman" Ihsan Igdifan Ali Al-Saree ${ }^{3}$ (D)}

${ }_{1,2,3}^{t, F a c u l t y}$ of Educational Sciences, Department of Psychological Sciences and Special Education/AlAlBayt University, Jordan.

Email:alkhateeb1512@yahoo.com

Email:Malak1942004@yahoo.com

'Email:ahs119193@yahoo.com

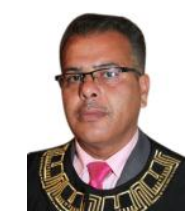

Corresponding Author

\section{Abstract}

The study examined the differences in the emotional intelligence levels among hearing-impaired and visually impaired students in Mafraq Governorate, Jordan. The study sample consists of 198 students; it is divided into 144 hearing-impaired and 54 visually impaired students who are enrolled in centers, schools, and institutions of special education of the academic year (2018-2019) in Jordan. To achieve the objectives of the study, the researcher developed a 38-item emotional intelligence scale in terms of four domains: Emotional knowledge, Emotional regulation, Empathy, and Social competence. The significance of validity and reliability is verified. The study results showed that the total degree of emotional intelligence among hearing-impaired students was low $(\mathrm{M}=1.51)$. The total degree of the emotional intelligence of the visually impaired students was medium $(\mathrm{M}=3.64)$. The results also revealed that there were statistically significant differences among hearing-impaired and visually impaired students in emotional intelligence as a whole and in all fields; the differences went in favor of visually-impaired students. The study recommended that the research and educational authorities, psychologists, and media officials should pay attention to individuals with auditory and visual disabilities and give them similar importance compared to normal people in terms of rights, facilities, values, and positive attitudes towards them.

Keywords: Emotional intelligence, Visual impairment, Hearing impairment, Jordan.

Citation | Akef Abdullah Al - Khateeb; Wael Mohammad Alshurman; Ihsan Igdifan Ali Al-Saree (2020). Emotional Intelligence Levels among Hearing-impaired and Visually Impaired Students in Jordan $7(4): 395-406$.

History:

Received: 4 September 2020

Revised: 25 September 2020

Accepted: 19 October 2020

Published: 2 November 2020

Licensed: This work is licensed under a Creative Commons

Attribution 3.0 License $(\boldsymbol{c c})$ EY

Publisher: Asian Online Journal Publishing Group
Acknowledgement: All authors contributed to the conception and design of the study.

Funding: This study received no specific financial support

Competing Interests: The authors declare that they have no conflict of interests.

Transparency: The authors confirm that the manuscript is an honest, accurate, and transparent account of the study was reported; that no vital features of the study have been omitted; and that any discrepancies from the study as planned have been explained.

Ethical: This study follows all ethical practices during writing.

\section{Contents}

1. Introduction

2. Literature Review .....................398

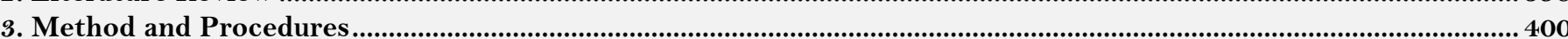

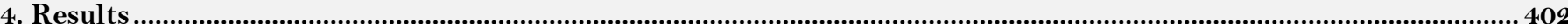

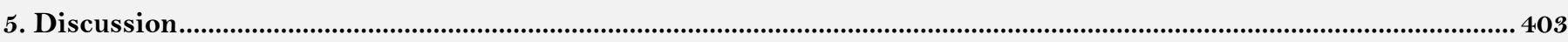

6. Conclusion …..................................... 404

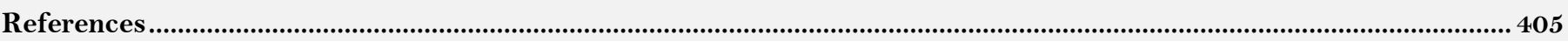




\section{Contribution of this paper to the literature}

This study contributes to the existing literature by determining the differences in the emotional intelligence levels among hearing-impaired and visually impaired students in Jordan.

\section{Introduction}

Emotional intelligence received great attention from researchers of various educational and psychological fields. This might be due to the growing interest in emotional intelligence; since it provides individuals with several opportunities for success in life domains and helps eliminate any misunderstanding. Emotional intelligence includes a group of social and moody features; they affect the diversity of emotional output of individuals in terms of their ability to face and comprehend problems. The more we understand feelings, the higher level we have in facing the individuals' daily-life problems. Pfeiffer (2001) considers emotional intelligence as one of the most modern types of intelligence; thus, it requires an unconventional approach to the concept of intelligence. Therefore, it is believed that the social, economic, health, environmental, cultural, and political changes facing society nowadays require not only mental abilities but also emotional abilities to be solved.

In his various books and articles, Goleman confirms that cognitive intelligence contributes with only (20\%) of the factors that determine success in life leaving $(80 \%)$ to factors other than the mental ones. The majority of those gaining high ranks are not distinguished due to their intelligence but rather to their possession of emotional intelligence skills Kurdi (2010). Bar-On believes that the people who possess emotional intelligence can recognize and express their emotions, understand the emotions of others, establish strong relations with them and take social responsibility without seeking to rely on others in their lives. Such individuals are typically optimists, happy, flexible, reliable, and successful in solving problems and in dealing with stress without losing control of themselves (Jarwan, 2012). Through emotional intelligence, one can successfully predict an individual's success in the practice patterns of life, in general, more than the Intelligence Quotient (IQ), while the general intelligence is a good predictor of success in the academic life. The lives of individuals are not only academic as they represent an aspect of the social life of the individual by nature. Individuals with high emotional intelligence are closer to success in any decision they make in their lives and work situations.

Emotional intelligence also contributes to building a person's character by making it more successful (Nofal, 2007). The Western systems and institutions focus on developing emotional skills of disabled people through psychological therapeutic intervention programs in educational institutions and centers of special education. As they aim at promoting self - esteem and emotional intelligence among students and their teachers seeking the best level of comprehensive social integration for all members of the community.

As for Arab societies, this goes through difficult stages when dealing with the category of people with disabilities in general and the category of people with visual and auditory disabilities. These difficulties may be attributed to teachers' negative attitudes toward people with visual and hearing impairments, or lack of experience and educational guidance which enable them to deal with those groups (Jarwan, 2012). The impact of the hearing and visual impairment is evident in various developmental aspects such as linguistic, psychological, social, cognitive, and mental growth and academic achievement. Such influences are evident in the adaptive behavior, which threatens the relations between family members and friends, since the adaptive behavior is an important factor incompatibility through reflection on intelligence and mental capacity.

Intelligence plays an effective role in improving personal and social abilities. The more intelligent the individual is, the more the individual can cope and adapt to the limitations of intelligence in some people with disabilities. So, their life becomes more complicated and they experience a sense of despair and distrust (Al-Imam \& Al-Jawaldh, 2016; Musa, 2009). In addition students with a visual and hearing impairment lack the proper adaptability in the interpersonal and social aspects and emotional problems such as isolation from peers, low selfesteem, translating the feelings and emotions of others, acute anxiety, and sudden surprise of their current abilities; they resort to facing the outside world with stubbornness and aggressiveness. Thus, they are often unable to take interpersonal and social responsibility as they will be characterized by the emotional fluctuation which affects their emotional and social development (Gardner \& Quallter, 2010).

The first person to use the term "Emotional Quotient" (EQ) versus the term "Intelligence Quotient" was BarOn, who sees emotional intelligence as a mixture or group of skills, traits, and social, personal, and emotional abilities that affect an individual's ability to adapt effectively to environmental pressures and requirements. According to the Bar-On's theory of emotional intelligence referred to in Reiff's Study (Reiff, 2001) we show the relationship of individuals with visual and auditory disabilities in line with the elements of emotional intelligence:

1. Social skills: Educational literature indicates that students with visual and hearing impairment have a deficiency in developing social relations and making new friendships. They also perform difficult tasks with the help of others. The results of the study by Mclntons, Vaughnn, and Zaragoza (Mayer \& Salovey, 2000) indicated that students with disabilities have a low level of emotional intelligence.

2. Interpersonal skills: Self - awareness is a key factor in developing interpersonal skills. Al-Saree, Alshurman, and Alshurfat (2020) showed the relationship between anxiety and self-esteem, and awareness and selfesteem in this category. The results showed that the high levels of awareness and self-esteem are directly linked with the low level of anxiety of people with disabilities.

3. Adaptability: studies indicate that the visually--impaired and hearing-impaired adults are less able to assess the sources of stress and anxiety. Moreover, Munsch' study reveals that university students suffer a lack of adaptability in university life (Mangal \& Mangal, 2004).

4. The ability to manage stress: Gregget's study reveals that impaired children and adults suffer from greater stress than their normal peers as they suffer from stress in the field of academic achievement (Aminpoor, 2013).

5. General mood: Goleman believes that working leads to optimism due to the circumstances in which we conduct better. It was clear that impaired children are incapable of learning to work and seem pessimistic towards the ways and methods of learning used for this category (Goleman, 1998). 
To sum up, students with hearing impairment need to organize and control themselves, develop academic requirements that help to solve problems and overcome difficulties, adapt to the new environment, develop effective compensatory skills and adopt strategies to support this category suffering from visual and hearing impairment.

\subsection{Problem of the Study}

The concept of emotional intelligence has received considerable attention from scientists during the last century due to its effect and prominent role in the psychology aspect of the individual and the learning process. Therefore, having high levels of emotional intelligence skills ensures the availability of more emotions and behaviors that reflect empathy, participation, assistance provision, and feeding others with sound ideas; those who enjoy empathy abilities tend to be less aggressive and conduct actions serving the community. The most important feature of emotional intelligence is that it is less than intelligence in terms of the genetic inheritance allowing parents, teachers, and special education specialists to develop the emotional intelligence for the impaired to determine the chances of individuals' success (Al-Khatib, 2014). Accordingly, it could be seen that emotional intelligence plays a major and active role in the mental health and psychological compatibility among individuals with visual and hearing impairments, leading to self-realization. Thus, higher levels of emotional intelligence among this group means the lesser personal problems and stress they face in life and the less the mental disorders one might face.

Individuals with hearing and visual impairment may feel anxiety and despair; so they became more likely to feel loneliness and develop the fear of the unknown, sensitivity, emotion, alienation, and low self - esteem. This leads to mental disorders. Also, the problem of the study comes from the researchers' interaction with individuals with visual and hearing impairments as they need more services, early intervention programs, and psychological counseling programs to interact positively with life and others. They can feel the loss of the real meaning of life more than others because of a disability and lack of efficiency resulted from visual and hearing impairments. Accordingly, this study is meant to be a research which has scientific and logic justifications after surveying the previous studies conducted on the emotional intelligence of people with visual and hearing impairments and the scarcity of studies on the levels of the emotional intelligence of people with visual and hearing impairments; thus, the study problem focuses on identifying the levels of the emotional intelligence of people with hearing and visual impairments.

\subsection{Questions of the Study}

1. What are the levels of emotional intelligence among the hearing-impaired students in Mafraq Governorate?

2. What are the levels of emotional intelligence among the visually impaired students in Mafraq Governorate?

3. Are there differences between the hearing-impaired and visually impaired students in terms of the levels of emotional intelligence?

\subsection{Importance of the Study}

The significance of this study stems from the role of emotional intelligence and its significant impact on forming of the students' personality, their understanding of themselves, and their interaction with the surrounding community, such as family and school in a positive manner.

The importance of this study can be seen in the following aspects:

1. This study provides tools to detect the levels of emotional intelligence among the hearing-impaired and visually-impaired students which help researchers carry out studies that contribute to providing scientific knowledge of different aspects to figure out differences between the emotional intelligence of the hearingimpaired and visually-impaired students.

2. The novelty of the study: it is related to identifying that hearing-impaired and visually impaired students possess emotional intelligence and stating the differences between the two disabilities.

3. The study will help specialists in psychological, guidance, educational, and social fields to provide possible support and assistance for this group of people with visual and hearing impairments.

\subsection{Study Limitations}

- This study was limited to assess the levels of emotional intelligence for the students enrolled in the schools and special education centers of people with visual and hearing impairments aged (12-17) in Mafraq Governorate.

- The study was also limited to the period when the study tools were applied during the academic year (20182019).

\subsection{Operational Definitions}

- Emotional Intelligence: the researcher adopts the operational definition of "Bar-On (2006) who defined it as an interaction of a group of emotional and social skills, competencies and facilitates affecting the individuals' ability to express and understand himself, understand and communicate with others, deal with his daily needs, and face challenges and stress (Bar-On, 2006).

- Visual impairment: the visually-impaired students are people who are officially diagnosed by Jordanian Diagnostic Centers as having a visual impairment and are enrolled in schools and special education centers in Mafraq Governorate in the academic year (2015 / 2016).

- Hearing impairment: the hearing-impaired students are people who are officially diagnosed by the Jordanian Diagnostic Centers as having a hearing impairment and are enrolled in schools and special education centers in Mafraq Governorate in the academic year (2015 / 2016). 


\section{Literature Review}

Nakpong and Chanchalor (2019) examined the use of interactive multimedia games in enhancing emotional intelligence among hearing-impaired students. The sample of the study consisted of (10) hearing-impaired students. Six interactive games were applied to develop emotional intelligence. To achieve the aim of the study, the experimental approach was used. The results of the study showed an effect of interactive games on the emotional intelligence of students with hearing disabilities on the dimensions: self-control, empathy, problem-solving, selfconsideration, and life satisfaction.

Diaz and Garcia (2018) aimed to identify the similarities and differences in the level of emotional intelligence, psychological flexibility, and self-esteem between ordinary individuals and individuals with disabilities. The sample of the study consisted of (110) normal people and people with disabilities. To achieve the aim of the study, a measure of emotional intelligence, psychological flexibility, and a measure of self-esteem were used. The results of the study showed that there are statistically significant differences in the level of emotional intelligence due to the variable of the study sample and for individuals with disabilities.

Eniola and Adebiyi (2007) which was conducted in Nigeria aimed at identifying the level of emotional intelligence and the ability to develop goals among a sample of students with visual impairment. The study also aimed to identify the effect of a counseling therapeutic program in improving emotional intelligence levels and the ability to develop career goals for this group of students. The sample of the study consisted of (32) male and female students with a visual disability who were randomly selected from one of the schools established especially for visual impairment students. Students participated in a counseling therapeutic program based on improving the level of motivation to know its impact on increasing the level of emotional intelligence and the ability to develop career goals. The study sample students responded to the Emotional Intelligence Scale developed by researchers and the ability to develop career goals before and after participating in the current study. The results of the study showed a significant effect of the counseling therapeutic program based on improving motivation levels in increasing the level of emotional intelligence and the ability to develop career goals among students with visual impairment.

Ataabadi, Yousefi, and Moradi (2013)'s study was conducted in Iran; it aimed to detect the level of emotional intelligence, social skills, family communication, self - esteem, and the prediction ability of these variables regarding the academic achievement among deaf students. The study sample consisted of 51 male deaf students and 55 female deaf students or hearing-impaired students who were randomly selected from some private schools in Tehran. To achieve the objectives of the study, Petrides and Furnham (2001) scale of the emotional intelligence was used in addition to the social skills, family communication, and self - esteem scales. The results of the study revealed that the level of emotional intelligence, social skills, family communication, and self - esteem among deaf students was low. The results showed there is a statistically significant positive correlation between emotional intelligence and family communication on one hand and the academic achievement of the deaf students on the other hand. The results also showed that there is no statistically significant correlation between social skills and academic achievement.

The study of Al-Khawaja (2013) aimed at analyzing the relationship between emotional intelligence and competitive strategies in civil society organizations in Jordan. To achieve the objective of the study, the researcher prepared a questionnaire consisting of (74) items including the elements of emotional intelligence, namely: (self vigilance, self - control, motivation, empathy, and social skills). The study sample consisted of (225) senior and middle management members. The study concluded that there were statistically significant differences between emotional intelligence and the strategy of cost leadership in Jordanian civil society organizations. There were no statistically significant differences in the perceptions of respondents about emotional intelligence due to the properties of the organization in terms of size, structure, and membership. Also, there were no statistically significant differences in the perceptions of respondents about competitive strategies due to the characteristics of the organization.

The study of Attri and Rai (2013) was conducted in India; it aimed to detect the level of emotional intelligence among visually-impaired students in public and inclusive schools. The sample of the study consisted of (120) male and female visually impaired students in regular and inclusive schools who were randomly chosen. To achieve the objectives of the study, a developed emotional intelligence scale was used by the researchers. The results revealed that the level of emotional intelligence among visually-impaired and normal students in both the regular and inclusive schools was medium and that there were no differences in the level of emotional intelligence among the visually-impaired due to gender.

The study of Mirzaei and Saeedi (2013) was conducted in Iran; it aimed to compare the level of emotional intelligence and the interpersonal characteristics between visually-impaired people and normal people. The study sample consisted of 18 men and 28 women who were selected randomly from Tehran City. To achieve the objectives of the study, the scale of the interpersonal characteristics was used in addition to (Bar-on) scale of emotional intelligence. The results showed that the level of emotional intelligence among the visually impaired people has ranged from low to medium while it was medium among normal people. The results showed that individuals with disabilities are characterized by openness while the normal students are characterized by neuroticism.

The study of Kumar and Singh (2013) was conducted in India; it aimed to detect the level of emotional intelligence and psychological compatibility between the visually-impaired students and normal students. The sample of the study consisted of (150) normal students selected intentionally from several primary and secondary schools. To achieve the objectives of the study, the compatibility scale among the students developed by Sinha and Singh (1993) was used in addition to Mangal and Mangal (2004) scale of emotional intelligence. The results of the study showed that the level of emotional intelligence and psychological compatibility among the visually impaired students was medium while the level of emotional intelligence and psychological compatibility among normal students ranged from medium to high. The findings also revealed that there was a positive correlation between emotional intelligence and psychological compatibility between students with a visual impairment and normal students and there were no differences in the level of emotional intelligence and psychological compatibility in favor of normal students. 
The study of Yasin, Bari, and Salubin (2012) was conducted in Malaysia; it aimed to detect the level of emotional intelligence among the deaf and hearing-impaired students. The sample of the study consisted of (148) male and female deaf and hearing-impaired students selected randomly from several private schools in Kuala Lumpur. To achieve the objectives of the study, the teacher's and parents' grading scale of measuring the level of emotional intelligence of the student was used. The results of the study showed that the level of emotional intelligence among the deaf and hearing-impaired students was medium. There were no differences in the level of emotional intelligence due to gender; there were differences in the level of emotional intelligence due to the grade level in favor of higher grades; moreover, there was a positive correlation between the level of emotional intelligence and academic achievement of students. Finally, there was a negative correlational between the high level of emotional intelligence and the behavioral problems among deaf and hearing-impaired students.

The study of Haghighatzade (2012) was conducted in Iran; it aimed to compare the level of emotional intelligence of deaf and normal students. The study sample consisted of (30) male and female deaf students and 30 normal students who were randomly selected from inclusive schools in Isfahan. To achieve the objectives of the study, the emotional intelligence scale was used in the data collection process. The results of the study revealed that the level of the emotional intelligence among the deaf students was medium, while it ranged from medium to high level among normal students; moreover, there were differences in the level of the emotional intelligence between deaf and normal students in favor of the normal students.

The study of Deluzio, Girolametto, and Schlauch (2011) was conducted in Canada; it aimed to identify the levels of social intelligence and social interaction with peers among a sample of hearing-impaired students. The study sample consisted of 12 hearing-impaired students and 12 normal students. Observation during play and the social intelligence scale were used in the process of collecting data from the study sample. The results revealed that there were differences in the level of social intelligence and social interaction with the peers between the hearing impaired students and normal students in favor of normal students. The results also showed that the level of social intelligence among the hearing - impaired students was low while the level of social intelligence among the normal students was medium.

The study of Vogel-Walcutt, Schatschneider, and Bowers (2011) was conducted in Florida; it aimed to identify the levels of social and emotional intelligence among a sample of the primary school hearing-impaired students. The study sample consisted of two groups, a group of 20 male and female hearing-impaired students and a group of 20 normal students who were randomly selected from some of the private and public schools. The emotional and intelligence scale of teachers' edition and observation were used in the process of collecting data from the study sample. The results revealed that there were differences in the level of the social and emotional intelligence between normal students and the hearing-impaired students in favor of the normal students.

The study of Chan (2008) studied the relationship between emotional intelligence, emotional maturity, leadership properties, and social relations of a sample consisting of (398) distinguished hearing-impaired students in Hong Kong. The study revealed that students with hearing impairment are more interested in leadership activities and the method of solving problems while they were less intelligent emotionally and less interested in social relations with others.

The study of Al-Ja'afreh (2007) aimed to detect the relationship between multiple intelligences in hearingimpaired children and the variables of the degrees of impairment, gender, and age (6-11 years) through a tool that has been developed containing ( 68 ) items including types of intelligences; the sample consisted of $(78):(42)$ males and (36) females. The results indicated there were statistically significant differences in favor of the relationship of the multiple intelligences among the study sample due to the degree of impairment and the differences were in favor the hearing-impaired people with a medium level; moreover, there were significant differences in favor of the relationship of the multiple intelligences due to the variable of age in favor of the group aged (4-6) years and significant differences in favor of the relationship of the multiple intelligences of the sample due to gender in favor of simple hearing-impaired females.

The study of Al-Ajami (2006) aimed to detect the differences between the study groups of clever and un clever blind female and male students in terms of academic achievement, interpersonal intelligence, social intelligence, and emotional intelligence. The sample consisted of 108 blind students (10-15 years) in GCC countries. Three scales of the interpersonal, social, and emotional intelligence were used as they were developed to achieve the objectives of the study. The results showed that there were significant differences in the interpersonal and emotional intelligence and all kinds of intelligence according to gender in favor of the males, significant differences in the emotional intelligence according to gender interaction and academic achievement in favor of males, significant differences in the emotional intelligence according to gender (females only) in favor of low female achievers and finally significant differences in emotional intelligence and all the kinds of intelligence per the academic achievement (clever students only) in favor of males.

The study of Sharma (2006) was conducted in India; it aimed to identify the level of the emotional stability and emotional intelligence of the visually-impaired students and their relationship with their school habits. The sample of the study consisted of (60) secondary school students who were visually impaired; the sample was randomly chosen. To achieve the objectives of the study, Attri and Rai (2013) scale for the emotional stability of children and (Brown \& Holtzman's) scale that was developed to the Indian environment were used. The results of the study revealed that the level of emotional stability among the visually-impaired students was medium and there was a statistically significant positive effect in terms of the high level of emotional stability among the visually-impaired on their positive habits.

The study of Austin (2006) aimed to identify emotional intelligence, personality, life satisfaction, social participation, social support, and its relationship to happiness and mental health. The study sample consisted of 500 Canadian and 204 Scottish students on whom the emotional intelligence scale, emotional participation, life satisfaction, social support, and public health were applied. The results showed that there was a positive relationship between emotional intelligence and the quality and size of the social relations, life satisfaction, and positive health and there were significant differences between the emotional intelligence, emotional participation and communication skills. Individuals with high intelligence can feel more in control of their ideas and more successful in academic performance and social field. 
The study of Dyck, Farugia, Schochet, and Holmes-Brown (2004) was conducted in Canada; it aimed to identify the differences in emotional and social intelligence levels between a sample of hearing-impaired students and a sample of visually-impaired students who were randomly selected from (4) private schools. The study sample consisted of (49) male and female students with a hearing impairment, (42) male and female students of the visually-impaired students in addition to a third group that is made up of (72) normal male and female students. The members of the study sample of the three groups responded to the scale of emotions distinguishing and the social communication scale. The study showed that there were differences in the level of social and emotional intelligence in favor of normal students compared with the hearing-impaired and visuallyimpaired students; moreover, there were differences in the level of social and emotional intelligence between hearing-impaired students and visually-impaired students in favor of the latter. After reviewing literature review, we conclude: Even though the previous studies that have been reviewed were applied to hearing-impaired and visually-impaired people, they are still considered normal people but they are derived from the gifts of sight and hearing; they share (with those who can hear and see) emotions, interpersonal traits, emotions and playing social roles. The role is shown by the previous studies regarding the relation of the emotional intelligence and its success in the individual's life in all psychological, physical and social aspects, the negative impact caused by the weakness of emotional intelligence of individuals and the negative psychological and social compatibility, interpersonally disorders and depression would contribute to highlighting the problem of the current study and the need to focus on the importance of the emotional intelligence for people with visual and hearing impairment. It was clear that the previous studies paid attention to the structure of the emotional and social intelligence for the special and normal categories such as the study of Diaz and Garcia (2018) and Al-Khawaja (2013) there were studies related to emotional and social intelligence of visually and hearing impairment like the study of Eniola. and Busari (2014); Yasin et al. (2012); Mirzaei and Saeedi (2013); Kumar and Singh (2013); Haghighatzade (2012); Deluzio et al. (2011); Vogel-Walcutt et al. (2011); Chan (2008); Al-Ajami (2006); Sharma (2006) and Dyck et al. (2004). This study goes in line with the previous studies regarding using the descriptive and analytical approach. This study also makes benefits from these previous studies in preparing the emotional intelligence scale for the students with hearing and visual impairment. This study differs from the previous in terms of identifying the differences in the levels of emotional intelligence among hearing-impaired students and their visually impaired peers in light of the variable of age.

\section{Method and Procedures}

This section discusses the procedures that were followed to reach the results of the study. It includes the study methodology that the researcher follows, the study and sample community, the study tool, the validity and reliability, and variables of the study.

\subsection{Methodology}

This study is classified as analytical descriptive research as it is based on the study and description of the educational phenomenon as found in real life and expressed in terms of quantity and quality (Al-Jadri \& Abu, 2009). This study aims to describe the levels of the emotional intelligence of hearing-impaired students and their visually impaired peers.

\subsection{Study Population}

The study population consists of all the hearing-impaired students and the visually impaired students enrolled in schools, institutions, and special education centers in Mafraq Governorate, Jordan. The total number of the sample is (198) male and female students chosen from two schools and four private education centers as shown in Table 1.

Table-1. Distribution of the study members of the visually- impaired and hearing-impaired students in Mafraq Directorate of Education.

\begin{tabular}{c|c|c}
\hline & Categories & Frequency \\
\hline Impairment & Hearing & 144 \\
\hline \multirow{2}{*}{} & Visual & 54 \\
\cline { 2 - 3 } & Total & 198 \\
\hline
\end{tabular}

\subsection{Study Sample}

The sample of the study consisted of all members of the study population including (198) male and female students: 144 hearing-impaired and 54 were visually impaired students. The visually impaired and hearingimpaired students were chosen intentionally by $100 \%$. There were (198) questionnaires distributed, and (192) were returned.

\subsection{Study Tool}

The researcher built a scale to collect the data of this study; the Emotional Intelligence Scale.

\subsubsection{Emotional Intelligence Scale}

An emotional intelligence scale consisting of (38) items was developed. The researcher used the 5-point Likert scale as follows: always (5), often (4), sometimes (3), rarely (2), and never (1).

\subsubsection{Scale Components}

This scale was developed through reviewing the theoretical literature on emotional intelligence and its skills, previous studies related to the variables of this study, such as: Al-Khawaja (2013); Al-Milli (2011); Al-Minshawi (2009); Alnawasrah (2008) and Al-Jundi (2006); Mayer, Salovey, and Caruso (2000) and Bar-On (2006). The scale 
of emotional intelligence was developed considering those studies. The scale of emotional intelligence, in its initial version, consisted of (48) items that were distributed in four fields: emotional knowledge, emotion regulation, empathy, and social competence.

\subsubsection{Scale Validity}

The initial version of the scale was verified to measure the purpose for which it was prepared through being presented to a group of ten arbitrators with academic and professional expertise in the field of special education psychology and counseling to figure out their opinions about the appropriate items of the domain and their clarity and to receive valuable appropriate notes. An agreement rate of $(80 \%)$ was adopted as a standard that determines the acceptance of the item. The most important amendments for the emotional intelligence scale were as follows: deleting (10) items from the scale and reformulating some items; so the final version of the scale consists of (38) items in instead of (48) items; they were distributed on four fields according to Table 2.

Table-2. Field and number of items in each field.

\begin{tabular}{c|l|c}
\hline Number & Field & Number of items \\
\hline 1. & Emotional knowledge & 10 \\
\hline 2. & emotion regulation & 10 \\
\hline 3. & Empathy & 9 \\
\hline 4. & Social competence & 9 \\
\hline \multicolumn{2}{c}{ Total } & 38 \\
\hline
\end{tabular}

\subsubsection{The Reliability of the Emotional Intelligence Scale}

The reliability was calculated through the (Test-Retest) method by applying the scale on a random prospective sample which consisted of (20) male and female students from a similar community to the study community in addition to non-sample students in the Northern Badia District, Mafraq Governorate; the sample consists of (10) visually-impaired male and female students and (10) hearing-impaired male and female students. The emotional intelligence scale was applied twice between them a time of two weeks and the reliability coefficients were calculated by using Cronbach's alpha to figure out the reliability of the internal consistency of the first test; the reliability coefficients were as shown in Table 3 :

Table-3. Internal consistency Cronbach's alpha and test-retest reliability of the fields and the total score (emotional intelligence scale).

\begin{tabular}{l|c|c}
\hline Field & Reliability of test-retest & Internal consistency \\
\hline Emotional knowledge & 0.92 & 0.92 \\
\hline Emotion regulation & 0.94 & 0.92 \\
\hline Empathy & 0.89 & 0.92 \\
\hline Social competence & 0.90 & 0.93 \\
\hline Emotional intelligence as a whole & 0.91 & 0.95 \\
\hline
\end{tabular}

Table 3 shows that the reliability coefficients of the test method of the fields of the emotional intelligence ranged from (0.89- 0.94), the reliability percentage of the emotional intelligence as a whole was (0.91), the reliability coefficients through the internal consistency ranged between (0.92-0.93), and the ratio of the internal consistency of the emotional intelligence as a whole was (0.95); all these were acceptable for the purposes of this study.

\subsubsection{Emotional Intelligence Scale Marking}

The Emotional Intelligence Scale in its final format consisted of (38) items and Likert scale (always, often, sometimes, rarely, never) was used to judge the levels of the visually-impaired and hearing-impaired students; the scale was marked by giving the previous scale the numbers (1, 2, 3, 4, 5); thus, the highest mark shall be (190) and the lowest mark shall be (38). To judge the emotional intelligence levels for the visually impaired and hearingimpaired students, the statistical criterion was used through the following equation: Category length= The highest limit - the lowest limit (progressive) 5-1

Number of suggested categories $=1.33$

- $1+1.33=2.33$ : the items with the arithmetic mean of (1-2.33) mean that the levels of the emotional intelligence among the visually impaired and hearing-impaired students was low.

- $2.34+1.33=3.67$ : the items with the arithmetic mean of (2.34-3.67) means that the levels of emotional intelligence among the visually impaired and hearing-impaired students was medium.

- $3.68+1.3=5$ : the items with the arithmetic mean of (3.68-5) means the levels of emotional intelligence among the visually impaired and hearing-impaired students was high.

\subsection{Statistical Analysis}

After processing the answers of the sample members, the researcher codified and processed them statistically using the (SPSS); some of the statistical processes include:

- Frequencies, arithmetic means, percentages, and standard deviations.

- (T-test).

- Cronbach's alpha equation to calculate the reliability coefficient. 


\section{Results}

4.1. To answer the first Question of the Study: "What are the Levels of the Emotional Intelligence among the Visually-Impaired and Hearing-Impaired Students in Mafraq Governorate?"

To answer this question, the arithmetic means, standard deviations, and the efficiency level of the emotional intelligence levels among the hearing-impaired students in Mafraq Governorate were calculated and designed in the descending order for all the dimensions of the tool as a whole; Table 4 shows that.

Table-4. Arithmetic means and standard deviations for the levels of the emotional intelligence among the hearingimpaired students in Mafraq Governorate in descending order for all the fields of the tool according to the

\begin{tabular}{|c|c|c|c|c|c|}
\hline Rank & N. & Domain & $\mathbf{M}$ & SD & Level \\
\hline 1 & 3 & Empathy & 1.94 & .564 & Low \\
\hline 2 & 4 & Social competence & 1.43 & .395 & Low \\
\hline 3 & 1 & Emotional knowledge & 1.39 & .351 & Low \\
\hline 3 & 2 & Emotion regulation & 1.37 & .326 & Low \\
\hline \multicolumn{3}{|c|}{ Emotional intelligence as a whole } & 1.51 & .312 & Low \\
\hline
\end{tabular}

Table 4 shows that the arithmetic means have ranged (1.37-1.94) where the field of empathy came in the first place with the highest arithmetic mean of (1.94) while the field of emotion regulation came in the last rank by the arithmetic mean of (1.37), and the arithmetic mean of the emotional intelligence as a whole was (1.51) by a low level of efficiency.

\subsection{The Results of the Second Question of the Study: "What are the Levels of Emotional Intelligence among the Visually-Impaired Students in Mafraq Governorate?"}

To answer this question, the arithmetic means, standard deviations and the level of the efficiency of the emotional intelligence levels among the visually-impaired students in Mafraq Governorate were calculated and designed in the descending order for all the dimensions of the tool as a whole; Table 5 illustrates this.

Table-5. Arithmetic means and standard deviations for the levels of emotional intelligence among the visually impaired students in Mafraqin the descending order for all the fields of the tool as a whole according to

\begin{tabular}{c|c|c|c|c|c} 
the arithmetic means. \\
\hline Rank & Number & Field & M & Standard Deviation & Level \\
\hline 1 & 4 & Social competence & 4.31 & .459 & High \\
\hline 2 & 3 & Empathy & 3.97 & .386 & High \\
\hline 3 & 1 & $\begin{array}{c}\text { Emotional } \\
\text { knowledge }\end{array}$ & 3.25 & .295 & Average \\
\hline 4 & 2 & Emotion regulation & 3.18 & .326 & Average \\
\hline \multicolumn{6}{c}{ Emotional intelligence. } \\
\hline
\end{tabular}

Table 6 shows that the arithmetic means have ranged (3.18-4.31) where the field of the social efficiency came in the first place with the highest arithmetic mean of (4.31) while the field of emotion regulation came in the last place with an arithmetic mean of (3.18), and the arithmetic mean of the emotional intelligence as a whole was (3. 64) by a medium efficiency level.

\subsection{The Results of the Third Question: Are There Differences between the Visually Impaired and the Hearing-} Impaired Students Regarding the Levels of Emotional Intelligence?

To answer this question, the arithmetic means, standard deviations for the levels of the emotional intelligence among the visually-impaired and hearing-impaired students were calculated; to figure out the statistical differences among the arithmetic means, T-test was used; Table 6 illustrates this process.

Table-6. Arithmetic means standard deviations and T-test among the visually and hearing-impaired students in the levels of emotional intelligence.

\begin{tabular}{|c|c|c|c|c|c|c|c|}
\hline & & No. & M & SD & "T" Value & $d f$ & Sig. \\
\hline \multirow[t]{2}{*}{ Emotional knowledge } & Hearing & 140 & 1.38 & .351 & -37.050 & 210 & .000 \\
\hline & Visual & 52 & 3.26 & .295 & & & \\
\hline \multirow[t]{2}{*}{ Emotion regulation } & Hearing & 140 & 1.38 & .326 & -36.311 & 210 & .000 \\
\hline & Visual & 52 & 3.18 & .326 & & & \\
\hline \multirow[t]{2}{*}{ Empathy } & Hearing & 140 & 1.94 & .564 & -25.677 & 210 & .000 \\
\hline & Visual & 52 & 3.97 & .386 & & & \\
\hline \multirow[t]{2}{*}{ Social competence } & Hearing & 140 & 1.43 & .395 & -45.808 & 210 & .000 \\
\hline & Visual & 52 & 4.31 & .459 & & & \\
\hline \multirow[t]{2}{*}{ Emotional intelligence as a whole } & Hearing & 140 & 1.52 & .312 & -46.461 & 210 & .000 \\
\hline & Visual & 52 & 3.65 & .278 & & & \\
\hline
\end{tabular}

Table 6 shows that there were no statistically significant differences at ( $\alpha=0.05$ ) among the hearing-impaired students and the visually-impaired students in all the fields and the total score of the emotional intelligence; the differences were in favor of the visually-impaired students. 


\section{Discussion}

\subsection{Discussion of the Results of the First Question: What are the Levels of Emotional Intelligence among the} Hearing-Impaired Students in Mafraq Governorate?

The results showed that the total score of the levels of emotional intelligence among the hearing-impaired students of the age group (13-18) in the private institutions and centers of Mafraq Directorate of Education was low; empathy was ranked first while emotion regulation was ranked last. These results are due to the lack of attention and a good understanding of emotions and feelings and differentiating between emotions and awareness of the relationship between thoughts, feelings and events, and the ability to control emotions and directing them towards positivity. The researcher believes that many hearing-impaired children fail to develop some mental and behavioral skills needed to understand and resolve interpersonal difficulties. These students stay isolated due to avoidance of complex attitudes and negative behaviors represented in problems of sending and receiving messages and they misunderstood their behavior showing a high degree of egocentrism leading to a poor capacity for social communication and interaction among people with hearing impairment. The study of Al-Khatib. (2014) confirmed that the hearing-impaired often integrate as a community with similar social skills; they have close social interaction making them tend to withdraw and isolation; this leads to social and psychosocial maturity delay and severe lack of self-esteem and poor capability to express himself which generates reactions toward others, a sense of failure, speed of excitability and poor social problems solving skills.

The emotional situations have a severe negative impact on the interpersonal and social levels and they can be translated into a clear increase in the degree of violence, despair, selfishness, relationship failure, a tendency towards individuality, and psychological unity (Goleman, 2006). This indicates that the high level of emotional intelligence provides people with hearing impairment with psychological and social compatibility with the social, family, and school conditions. The high level of motivation affects increasing the level of emotional intelligence and the ability to set the functional goals; the lack of social participation and communication and the low level of self - esteem and the psychological health status have the greatest impact on their academic, social, communicative and professional lives in dealing with the demands of life. The researcher attributes this result to a lack of awareness and guidance programs provided to students with hearing impairment either from the family, teachers, specialists, educational institutions, or special education centers. The society's perception has a significant impact on this category; thus, the limited programs, recreational services, sports activities, and cultural and scientific competitions generate a great sense of loneliness and decrease the level of self- esteem and social skills and communication with others.

The studies of Austin (2006); Ataabadi et al. (2013); Deluzio et al. (2011) and Vogel-Walcutt et al. (2011) confirm the same results since they indicated a low level of the emotional intelligence of the hearing - impaired students; hearing - impaired students with the high level of intelligence had more control on their ideas and more compatibility in social participation, size of relations, communication skills and family communication on the one hand and the academic achievement on the other. Finally, the level of intelligence of hearing-impaired students was low.

This study doesn't go in line with the study of Ataabadi et al. (2013) which indicated that the level of the emotional intelligence and social skills among deaf and hearing-impaired students was medium. There was a statistically significant relationship between family communication on the one hand and emotional intelligence, social skills, and self-esteem on the other hand. The results of the study of Yasin et al. (2012) indicated that the level of emotional intelligence was medium for the deaf and hearing-impaired students, and there was a positive correlation between the level of the emotional intelligence and the academic achievement. There was a negative correlation between the high level of emotional intelligence and behavioral problems. The results of the study of Haghighatzade (2012) indicated that the level of the emotional intelligence among deaf students was medium. The study of Nakpong and Chanchalor (2019) whose results showed that there is an effect of interactive games on the emotional intelligence of students with hearing impairments on the dimensions of emotional control, empathy, problem-solving, self-esteem, and life satisfaction.

\subsection{Discussion of the Results of the Second Question: What are The Levels of Emotional Intelligence among the Visually Impaired Students in Mafraq Governorate?}

The results of this question indicated that the levels of emotional intelligence among the visually impaired students in Mafraq directorate of education and Special Education Centers in Jordan were medium. The researcher explains that the visually-impaired people obtain an average degree on the emotional intelligence scale due to the characteristics of this category as they suffer from many psychological problems, self-centeredness, lack of selfconfidence, poor adaptation, introverted ness, anxiety, tension, lack of social skills, alienation, and dependence. Thus, these studies have confirmed the same results. The researcher believes that poor adaptability and poor social skills are due to the category's feeling that restrictions are limiting their freedom so they cannot do what they want; they lack visual experience so they cannot enjoy movement since they lack the sense of sight; they cannot control the surrounding environment; they cannot act correctly in social situations, and they cannot imitate social behaviors or understand non-verbal forms.

This result can be attributed to the awareness of the visually-impaired students with their emotions and controlling them, getting rid of the negative emotions, motivating themselves in a way that enables them to achieve their goals and ability of emotional participation, management of social relations, a realization of different emotions, and differentiating between positive and negative emotions. They have the skills of the emotional participation and communication with others; they have more understanding of themselves and the surrounding environment; they are more able to express their emotions and self - satisfaction; thus, they can make friendships and relationships with others; this leads to increase their abilities to solve the problems they face and increase their desires in interacting with others.

The researcher believes that the visually impaired people's feelings were ignored and rejected by their families and the community; this leads to getting low degrees on the emotional intelligence scale. The researchers believe that the possibility of increasing the academic achievement of people with a visual impairment depends on their emotional and social skills; this can be accomplished via raising self - awareness, understanding, solving problems, managing emotions in the learning environment of teachers, students, and principals to pay attention to the 
emotional intelligence at schools and centers. The study of Goleman (2006) stresses that accessing to success starts with the mental ability but humans need social and emotional efficiency to make benefit from their mental and cognitive abilities. The reason behind the deficit of making benefit from mental and cognitive abilities is the absence of emotional and social efficiency. Goleman states that cognitive intelligence contributes by $20 \%$ at maximum only in the individual's success in life while other factors such as emotional intelligence contribute by 80\% (Zidane \& Al-Imam, 2002). This result was in line with the results of the study of Eniola. and Busari (2014); Attri and Rai (2013); Mirzaei and Saeedi (2013); Kumar and Singh (2013) and Sharma (2006) as they indicated that there was an average level of emotional intelligence of visually-impaired students. According to the researcher, the results of this study go in line with any other study.

\subsection{Discussion of the Results of the Third Question: Are there Differences between the Hearing-Impaired and the Visually Impaired Students in Terms of the Levels of Emotional Intelligence?}

The results of the study revealed there were statistically significant differences between the hearing-impaired students and the visually impaired students in Mafraq directorate of education and the special education centers in all fields and the emotional intelligence as a whole. The differences were in favor of the visually impaired students. The researcher attributes these results to the fact that the visually-impaired students enjoy all the characteristics enjoyed by normal students if surrounded by appropriate environmental conditions to develop their abilities and skills, so they respond to this development just like normal people; the study of Smith (2006) showed that the characteristics that have been observed in the visually-impaired students such as lack of self-esteem or lack of social maturity; withdrawal and negativity are the result because of the way through which the society deals with them.

The studies of Chan (2008); Al-Hadidi (2009)and Halawah (2007) state that all that needed by the hearingimpaired and visually-impaired students are providing appropriate opportunities for growth, independence, getting away from being deprived of opportunities of depending on themselves and dealing with them in terms of their weakness and disability; they should be provided with opportunities to compete and feel successful; in case of failure, it would be hard for them to develop positive and realistic relation with normal people. The visuallyimpaired people's freedom of practicing different skills with a little help is considered the basis of facing normal people and interact with others. The differences can be justified in favor of the visually impaired people since they are provided with opportunities to develop their abilities to which they respond. This study showed that people with visual impairment have adequate potential which enables them to develop their skills and abilities to the extent that makes them superior to their peers with hearing impairment. Their impairment is not an obstacle anymore; they can use the rest of their senses to make up for blindness by training and qualifying them to use other senses well and giving them opportunities to compete and feel successful. Suffering, disorders, emotional and social problems are the result of negative attitudes imposed by people towards them; so, the suffering goes back to these attitudes rather than their visual impairment.

The study of Alqraiti (2007) stresses that the negative attitudes towards people with the visual impairment affect adversely their characters because of negligence, lack of acceptance or excessive protection by those around them; this excessive protection makes them feel powerless to confront a lot of situations and weaken their self confidence leading to frustration which negatively affects their social relations and leads to withdrawal and introversion and eventually reaching to poor interpersonal and social compatibility.

The studies of Smith (2006); Sharma (2006) and Eniola and Adebiyi (2007) stresses the same results. These findings came as a result of the family and school environment and the social relations with others; moreover, the hearing-impaired people are less mature socially than others; they neglect the feelings of others, misunderstand their behavior and show a high degree of egocentrism; however, they are more interested in the leadership activities and the method of solving problems but they are less intelligent emotionally and more interested in the social-communicative relations with others; accordingly, the study of Chan (2008) affirms the same results. The results of this study go in line with the study of Haghighatzade (2012) which revealed that there were differences between deaf students and normal students in the levels of the emotional intelligence in favor of the normal students. This study was in line with Dyck et al. (2004) which showed that there were differences in the level of the emotional and social intelligence in favor of the normal students compared with the hearingimpaired and visually-impaired students and there were differences in the level of the social and emotional intelligence among students of hearing and visual impairments in favor of students with visual impairment. The study of Diaz and Garcia (2018) showed that there are statistically significant differences in the level of emotional intelligence due to the variable of the study sample and in favor of individuals with disabilities. This study wasn't in line with the study of Al-Ja'afreh (2007) whose results revealed that there were statistically significant differences for the relationship of multiple intelligences which includes the emotional intelligence that is attributed to the variable of the degree of impairment and the differences were in favor of the people with simple and medium hearing impairment and the group aged (4-6 years). The results of this study wasn't in line with the study of Yasin et al. (2012) which showed that the level of the emotional intelligence among students with hearing impairment was medium and there was a negative correlation between the high level of the emotional intelligence and the behavioral problems among students with hearing impairment.

The results of the study of Kumar and Singh (2013) showed that there is a positive correlation between emotional intelligence and the psychological compatibility among the students with visual impairment and normal students. The study of Al-Ajami (2006) showed that there were statistically significant differences in the interpersonal, emotional, and social intelligence according to gender interaction and academic achievement in favor of males.

\section{Conclusion}

The research and academic bodies, psychologists, and media must pay attention to the hearing-impaired and visually impaired people and they give them similar attention compared with normal people. Supporting and empowering the family to take responsibility for taking care of children, increasing the effectiveness of this role to make the children feel safe, comfortable, and secure, encouraging them to rely on themselves, allowing them to 
express their feelings freely and working on integrating them into society. Expanding family and school counseling to help society to accept people with impairment, help them to solve their psychological and social problems, raise their level of self - esteem with the planning and implementing mental health programs, and provide preventive services and health care. Studying the effect of emotional intelligence on the academic success of people with visual and hearing impairments. Studying the effect of emotional intelligence on predicting the level of life satisfaction in normal individuals and people with visual and hearing impairments.

\section{References}

Al-Ajami, M. (2006). Differences in the interpersonal, social, and emotional intelligence among the distinguished blind students and low achievers in the Arab Gulf Cooperation Council countries. Unpublished PhD Dissertation, Amman Arab University, Jordan.

Al-Hadidi, M. (2009). Introduction to visual impairment. Amman: Al-Fikr House of Publication and Distribution.

Al-Imam, M., \& Al-Jawaldh, F. (2016). Behaviors indicating the theory of mind. Amman, Jordan: The House of Culture.

Al-Ja'afreh, S. (2007). The relationship between multiple intelligences of the hearing impaired students and the variables of the degrees of impairment, gender, and age. Unpublished Master Thesis, Amman Arab University, Jordan.

Al-Jadri, A., \& Abu, H. Y. (2009). The methodological foundations and statistical uses in the educational and human sciences research. Amman: Esra for Publication and Distribution.

Al-Jundi, G. (2006). Differences in the emotional intelligence between gifted students and ordinary students and the relationship with academic achievement. Unpublished Master Thesis, Amman Arab University, Jordan.

Al-Khatib, J. (2014). Introduction to hearing impairment. Amman, Jordan: Al-Fiqr House for Publication and Distribution.

Al-Khatib., A. (2014). Quality control and accreditation in special education. Irbid, Jordan: Modern Book World for Publishing and Distribution.

Al-Khawaja, L. (2013). Analyzing the relationship between emotional intelligence and the competitive strategies in civil society organizations. Unpublished Master Thesis, Amman Arab University, Jordan.

Al-Milli, S. (2011). Differences in the emotional intelligence among a sample of outstanding and ordinary students: A field study on the first secondary students in the city of Damascus. Damascus University Journal, 2(1+2), 283-315.

Al-Minshawi, S. (2009). The relationship between happiness and emotional intelligence among a sample of Jordanian adolescents. Unpublished Master Thesis, Amman Arab University, Jordan.

Al-Saree, I. I. A., Alshurman, W. M., \& Alshurfat, S. S. (2020). The effectiveness of supervision in solving problems facing teachers of students with Autism Spectrum Disorder in Jordan. Journal of Education and E-Learning Research, 7(3), 314-322. Available at: https://doi.org/10.20448/journal.509.2020.73.314.322.

Alnawasrah, F. (2008). Emotional, social, and moral intelligence among talented students and their relationship to some demographic variables. Unpublished PhD Dissertation, Amman Arab University, Jordan.

Alqraiti, A. (2007). The psychology and education of people with special needs. Cairo: Al-Fiqr Al-Arabi House for Publication.

Aminpoor, H. (2013). Relationship between social intelligence and happiness in payamenoor university students. Annals of Biological Research, $4(5), 165-168$.

Ataabadi, S., Yousefi, Z., \& Moradi, A. (2013). Investigation of the multiple relations between emotional intelligence, social skills, and selfesteem with family communications among deaf and hard of hearing adolescents. International Research Journal of Applied and Basic Sciences, 3(11), 1600-1608.

Attri, A., \& Rai, B. (2013). Emotional intelligence of visually impaired adolescents studying in inclusive and exclusive settings. Educational Research International, 1(2), 33-40.

Austin, E. (2006). Emotional intelligence and emotional informational processing. Journal of Interpersonal and Individual Differences, 39, 403414.

Bar-On, R. (2006). The impact of emotional intelligence on subjective well-being. Interpersonal in Education, $23(2), 41-62$.

Chan, D. W. (2008). Giftedness of Chinese students in Hong Kong: Perspectives from different conceptions of intelligences. Gifted child quarterly, 52(1), 40-54. Available at: https://doi.org/10.1177/0016986207311058.

Deluzio, J., Girolametto, L., \& Schlauch, R. (2011). Peer interactions of preschool children with and without hearing loss. Journal of Speech, Language \& Hearing Research, 54(4), 1197-1210. Available at: https://doi.org/10.1044/1092-4388(2010/10-0099.

Diaz, M., \& Garcia, M. (2018). Emotional intelligence, resilience, and self-esteem in disabled and non-disabled people. Enfermeria Global, 17(2), 263-273. Available at: https://doi.org/10.6018/eglobal.17.2.291381

Dyck, M., Farugia, C., Schochet, L., \& Holmes-Brown, M. (2004). Emotion recognition/understanding ability in hearing or vision-impaired children: Do sounds, Sights, or words make the difference? Journal of Child Psychology and Psychiatry, 45(4), 789-800. Available at: 10.1111/j.1469-7610.2004.00272.x.

Eniola, M., \& Adebiyi, K. (2007). Emotional intelligence and goal setting - an investigation into interventions to increase motivation to work among visually impaired students in Nigeria. The British Journal of Visual Impairment, 25(3), 249-253. Available at: https://doi.org/10.1177/0264619607079805.

Eniola., S., \& Busari, A. (2014). Emotional intelligence in promoting self-efficacy of the visually impaired fresh students of federal college of education (special) Oyo, Nigeria. International Journal of Humanities and Social Science, 4(14), 170-179.

Gardner, K., \& Quallter, P. (2010). Concurrent and incremental Validity of three trait emotional intelligence measures. Australia Journal of Psychology, 62, 5-13. Available at: https://doi.org/10.1080/00049530903312857.

Goleman, D. (1998). Working with emotional intelligence. New York: Bantam Books.

Goleman, D. (2006). Social intelligence the new science of human relationships. New York: Bantam Dell.

Haghighatzade, R. (2012). A comparison of the emotional intelligence of two groups of deaf and non-deaf students in Isfahan. Journal of Basic and Applied, 2(9), 9573-9576.

Halawah, M. (2007). The effectiveness of a proposed suggested program for the development of emotional maturity in improving the social efficiency among a sample of hearing-impaired students emotionally abused. Unpublished PhD Dissertation, University of Alexandria, Egypt.

Jarwan, F. (2012). Emotional intelligence and emotional, social education. Amman: Al-Fikr House of Publication: Publishers \& Distributors.

Kumar, S., \& Singh, J. (2013). Emotional intelligence and adjustment among visually impaired and sighted school students. Asian Journal of Multidimensional Research, 2(8), 1-8.

Kurdi, S. (2010). Emotional intelligence and its relation to social skills, self-efficacy, and educational leadership among a sample of female principals of secondary schools in the western region of Saudi Arabia. Journal of Educational Sciences, 1, 123-144.

Mangal, S. K., \& Mangal, S. (2004). Mangal emotional intelligence inventory. Agra: National Psychological Corporation.

Mayer, J., \& Salovey, C. (2000). Emotional intelligence meet traditional standards for intelligence. Intelligence, 27(4), $267-298$.

Mayer, J., Salovey, P., \& Caruso, D. (2000). Models of emotional intelligence. In R. Sternberg (Ed.), Handbook of Intelligence (pp. 396-420). Cambridge: Cambridge University Press.

Mirzaei, S., \& Saeedi, R. (2013). Comparison of emotional intelligence and interpersonally traits of the successful and normal individual with visual impairments in Tehran city. International Journal of Applied Psychology, 3(3), 83-87. Available at: https://doi:10.5923/j.ijap.20130303.08.

Musa, R. (2009). Psychology of the hearing impaired. Jordan: Books World House for Publishing and Distribution.

Nakpong, N., \& Chanchalor, S. (2019). Interactive multimedia games to enhance the emotional intelligence of deaf and hard of hearing adolescents. International Journal of Instruction, 12(2), 305-320. Available at: 10.29333/iji.2019.12220a.

Nofal, B. (2007). Multiple intelligences in the classroom: Theory and practice. Amman: Al-Masira House for Publication and Distribution.

Petrides, K. V., \& Furnham, A. (2001). Trait emotional intelligence: Psychometric investigation with reference to established trait taxonomies. European Journal of Personality, 15(6), 425-448. Available at: https://doi.org/10.1002/per.416.

Pfeiffer, S. (2001). Emotional Intelligence: Popular but elusive construct. Report Review, 23(3), 138-142. Available at: https://doi.org/10.1080/02783190109554085. 
Reiff, H. (2001). The relation of LD and gender with emotional intelligence in college students. Journal of Learning Impairment, 34(1), 66-78. Available at: https://doi.org/10.1177/002221940103400106.

Sharma, S. (2006). Emotional reliability of visually impaired in relation to their study habits. Journal of the Indian Academy of Applied Psychology, 32(1), 30-32.

Sinha, A., \& Singh, B. (1993). Manual for adjustment inventory for school students. Agra: Natinal Psychological Corporation.

Smith, D. (2006). Introduction to special education: Teaching in an age of opportunity (5th ed.). Boston: PearsonEducation, Inc

Vogel-Walcutt, J. J., Schatschneider, C., \& Bowers, C. (2011). Social-emotional functioning of elementary-age deaf children: A profile analysis. American Annals of the Deaf, 156(1), 6-22. Available at: https://doi.org/10.1353/aad.2011.0017.

Yasin, M., Bari, S., \& Salubin, R. (2012). Emotional intelligence among deaf and hard of hearing children. The Social Sciences, 7(5), 679-680.

Zidane, E., \& Al-Imam, K. (2002). Emotional intelligence and its relationship to methodsEducation and some dimensions of personality among students of the Faculty of Specific Education. Research JournalEducational and psychological, Menoufia University, Egypt, 17(3), $1-41$. 